\title{
Orthotopic Ileal Neobladder Reconstruction for Bladder Cancer: Is Adjuvant Chemotherapy Safe?
}

\author{
Murugesan Manoharan, Martha A. Reyes, Rakesh Singal, Bruce R. Kava, Alan M. Nieder, \\ Mark S. Soloway
}

Department of Urology and Department of Medicine (RS), University of Miami School of Medicine, Miami, Florida, USA

\begin{abstract}
Objective: We examined our database of patients undergoing radical cystectomy (RC) with orthotopic neobladder (NB) to determine whether adjuvant chemotherapy in this group is safe.

Materials and Methods: We performed a retrospective analysis of patients who underwent radical cystectomy and urinary diversion between 1992 and 2004. Relevant clinical and therapeutic data were entered into a database. High-risk bladder cancer patients who underwent NB were identified. They were stratified into 2 groups, those who received adjuvant chemotherapy and those who did not. The incidence of complications between the 2 groups was analyzed and compared. Results: Over the 12-year period, 136 patients underwent RC and NB construction for bladder cancer. Of these, 83 patients were at high risk for recurrence. Nineteen patients received adjuvant chemotherapy and 64 did not. The complication rate in the adjuvant chemotherapy group was $53 \%$ and it was $23 \%$ in those who did not receive chemotherapy. There were no perioperative or treatment related death. There were 2 patients with grade 4 toxicity in the adjuvant chemotherapy group. There was a statistical difference between these two groups with regard to the incidence of complications. However, none of these complications was life-threatening, required only conservative treatment and caused no long-term disability.

Conclusions: Adjuvant chemotherapy is a safe treatment for patients undergoing RC and NB substitution. Hence, the option of orthotopic NB should not be denied in selected bladder cancer patients with high risk for recurrent disease.
\end{abstract}

Key words: bladder neoplasms; urinary diversion; chemotherapy

Int Braz J Urol. 2006; 32: 529-35

\section{INTRODUCTION}

38,000 men and 15,000 women are newly diagnosed with bladder cancer annually in the United States and about 15000 are expected to die from this each year (1). A common therapeutic modality for treating muscle invasive bladder cancer is radical cystectomy (RC) with the reconstruction of a urinary diversion. Over the last 15 years, orthotopic neobladder
(NB) reconstruction has become a recognized method of urinary diversion in properly selected bladder cancer patients as the NB offers quality of life advantages with no significant difference in morbidity in relation to other types of urinary diversion such as ileal conduit (2-4). Other advantages of a neobladder include the achievement of a low-pressure reservoir, maintenance of near-normal volitional voiding and the avoidance of an external stoma. 
Currently patients at high risk for recurrent disease, such as clinical stage $>\mathrm{T} 2$ with or with out positive lymph nodes are frequently offered adjuvant chemotherapy (AC). Studies have demonstrated that $\mathrm{AC}$ in these situations have a positive clinical impact and improves survival (5-7). Even in these high-risk patients, NB reconstruction is increasingly being offered as a method of choice for urinary diversion. The incidence of urinary tract infections, the need for intermittent self-catheterization, incomplete emptying of bladder and mucus plugs are common problems associated with a NB reconstruction. Chemotherapy is associated with significant toxicity such as neutropenia and immunosupression resulting in increased incidence of complications including sepsis (8). Consequently, concern arises whether the NB patients can tolerate the AC safely.

There are unanswered questions about the complication rate related to adjuvant chemotherapy in a NB setting. There is paucity of information in the current literature regarding the incidence of complications in patients with a NB who received AC compared to those who did not receive AC. Hence, we analyzed two groups of our NB patients, those who received adjuvant chemotherapy and those who did not, comparing the complications to determine whether adjuvant chemotherapy is safe in patients undergoing $\mathrm{RC}$ with $\mathrm{NB}$.

\section{MATERIALS AND METHODS}

A retrospective analysis was performed on patients with bladder cancer treated with radical cystectomy and neobladder reconstruction between 1992 and 2004 at the University of Miami. After obtaining institutional review board approval, relevant clinical and therapeutic data were entered into a database. Following a standard radical cystectomy and pelvic lymph node dissection, a neobladder reconstruction was performed using modification of the technique described by Hautmann \& Studer $(9,10)$. Patients at high risk for recurrent disease following $\mathrm{RC}$ such as clinical stage $>\mathrm{T} 2$, nodal disease and/ or metastatic disease were referred to the oncologist for considering adjuvant chemotherapy. The final decision to administer $\mathrm{AC}$ was made by the oncologist in conjunction with the patient's desires.

The patients were stratified into 2 groups, those who received adjuvant chemotherapy and those who did not. Adjuvant chemotherapy protocols were administered at the discretion of the medical oncologist. The incidence of urinary tract infection, gastrointestinal and hematologic complications, wound infections and other complications between these 2 groups were analyzed and compared. The toxicity of chemotherapy in the group who received adjuvant chemotherapy was scored in accordance to the National Cancer Institute Common Toxicity Criteria (NCI CTC) and recorded in to the database. The chi-square test was used to assess statistical significance in complication rates between the groups, ( $\mathrm{p} \leq 0.05$ considered statistically significant).

\section{RESULTS}

Over a 12 year period, 136 patients underwent radical cystectomy and neobladder reconstruction for bladder cancer at our center. 129 (95\%) were males and $7(5 \%)$ were females with a mean age of 66 years. $83(61 \%)$ patients were at high risk for recurrence and had clinical stage $>\mathrm{T} 2$, nodal disease and/ or metastatic disease. In this group, 19 patients received adjuvant chemotherapy (Group-1) and 64 did not (Group-2). Table-1 shows the base line characteristics and the pathologic stage of the study groups. The distribution of chemotherapy regimens are detailed in Table-2. The complications are listed in Table-3 and toxicity by grade in group 1 is shown in Table- 4 .

Overall, 25 patients (29\%) suffered a complication: 7 (29\%) patients had urinary tract infections; 7 (21\%) had gastrointestinal complications; 8 (33\%) had hematologic manifestations; 2 (8\%) had wound infection and $10(12 \%)$ had miscellaneous complications. In miscellaneous complications one patient from Group-1 had to change chemotherapy regimens from gemcitabine and cisplatin to paclitaxel and carboplatin because of renal impairment; two patients had poor appetite and loss of weight. From Group-2, two patients had cardiac complications (severe chest pain with atrial fibrillation on 
Table 1 - Base-line characteristics of the patients.

\begin{tabular}{lcc}
\hline Characteristics & $\begin{array}{c}\text { NB with AC } \\
\text { Group 1 }\end{array}$ & $\begin{array}{c}\text { NB without AC } \\
\text { Group 2 }\end{array}$ \\
\hline N. of patients & 19 & 64 \\
Sex (M/F) & $17 / 2$ & $62 / 2$ \\
Mean Age (years) & 66 & 66 \\
Mean Follow up (months) & 34 & 29 \\
Pathological Stage & & \\
T2 & 6 & 30 \\
T3 & 9 & 28 \\
T4 & 4 & 6 \\
N0 & 4 & 54 \\
N1 & 5 & 4 \\
N2 & 10 & 5 \\
Nx & 0 & 1 \\
M0 & 18 & 62 \\
M1 & 1 & 1 \\
Mx & 0 & 1 \\
\hline$N$
\end{tabular}

$N B=$ neobladder $; A C=$ adjuvant chemotherapy.

electrocardiogram, and myocardial infarction), two patients had wound dehiscence, one patient had pouchitis requiring intravenous antibiotics, one patient had an entero-neobladder fistula that required repair and one had a pelvic abscess that required CT guided drainage. Complications were demonstrated in $10(53 \%)$ patients from Group-1 and $15(23 \%)$ patients from Group-2 ( $\mathrm{p}<0.01)$. As expected, hematologic complications were the most frequent to occur in Group-1. Three patients had anemia, 3 patients had neutropenic fever, and 2 patients had

Table 3 - Complications.

\begin{tabular}{lllr}
\hline & $\begin{array}{c}\text { Group-1, } \\
\text { N (\%) }\end{array}$ & $\begin{array}{c}\text { Group-2, } \\
\text { N (\%) }\end{array}$ & p Value \\
\hline Urinary tract infection & $2(11)$ & $5(8)$ & 0.70 \\
Gastrointestinal & $5(26)$ & $2(3)$ & $<0.01$ \\
Hematologic & $8(42)$ & 0 & $<0.01$ \\
Wound infection & $0(0)$ & $2(3)$ & 0.43 \\
Others & $3(16)$ & $7(11)$ & 0.56 \\
\hline
\end{tabular}

Table 2 - Distribution of chemotherapy regimens in group-1.

\begin{tabular}{lc}
\hline Chemotherapy & N. of Patients \\
\hline Methotrexate, vinblastine, & \\
$\quad$ doxorubicin \& cisplatin (MVAC) & 9 \\
Paclitaxel \& carboplatin & 3 \\
Gemcitabine \& platinum & 6 \\
Carboplatin \& etoposide & 1 \\
Total & 19 \\
\hline
\end{tabular}

anemia and neutropenic fever. Urinary tract infection was the most frequent complication in Group-2. There were no perioperative or treatment related deaths. Although the rate of hematologic complications in Group-1 was significant, these complications were not life-threatening, required only conservative treatment, and there were no long term sequelae. Additionally symptoms were transient and resolved when chemotherapy was completed.

Table 4 -Distribution of chemotherapy toxicity by grades according to the National Cancer Institute Common Toxicity Criteria.

\begin{aligned} \hline Toxicity Type & Group $\mathbf{1}(\mathbf{n}=\mathbf{1 9}) \\ & \mathbf{N}(\boldsymbol{\%}) \\$\hline Gastrointestinal & \\ Grade 1 & $4(21) \\$ Grade 2 & $1(5) \\$ Grade 3 & $0(0) \\$ Grade 4 & $0(0) \\$ Hematologic & $3(16) \\$ Grade 1 & $0(0) \\$ Grade 2 & $3(16) \\$ Grade 3 & $2(11) \\$ Grade 4 & \\ Other & $2(11) \\$ Grade 1 & $1(5) \\$ Grade 2 & $0(0) \\$ Grade 3 & $0(0) \\$ Grade 4 & \end{aligned}




\section{COMMENTS}

In recent years, the neobladder has become the preferred form of bladder reconstruction in a selected patient population after radical cystectomy (2). The neobladder has replaced ileal conduit as the standard procedure due to better quality of life outcomes, avoidance of stoma, an improved body image and no additional morbidity $(11,12)$. In addition, neobladder reconstruction does not compromise the cancer control.

Despite these advantages there are some disadvantages with the reconstruction of a neobladder. Some neobladder patients do not have the normal neuromuscular functions needed for micturition as voiding depends on a satisfactory relaxation of the pelvic floor and increase in the abdominal pressure. Therefore some patients with voiding dysfunction will require clean intermittent catheterization in order to empty the neobladder completely and to avoid urinary tract infections (13). During the immediate post operative weeks, patients are often required to catheterize to irrigate the bladder to remove the mucus.

Wood et al. (14) prospectively evaluated 66 patients with neobladder and found that $26(39 \%)$ and $8(12 \%)$ patients had a urinary tract infection and urosepsis, respectively. This study demonstrated that the estimated 5-year probability of urinary tract infection and urosepsis for patients who voided independently were $58 \%$ and $18 \%$, respectively and that the only factors predictive of urinary tract infection on multivariate analysis were urine culture with greater than 100,000 cfu bacteria and female gender. Also, recurrent urinary tract infection was the only predictor for urosepsis and intermittent catheterization or hydronephrosis was not related to urinary tract infection or urosepsis.

Despite the improvements in postoperative care and surgical techniques, approximately $50 \%$ of patients who undergo cystectomy can expect disease recurrence, which may require adjuvant chemotherapy. Many studies have reported that adjuvant chemotherapy gives an improved diseasefree survival over radical cystectomy alone for extravesical bladder cancer $(15,16)$. Susuki et al. (17) demonstrated in a retrospective study that adjuvant chemotherapy with methotrexate, vinblastine, doxorubicin and cisplatin (MVAC) and methotrexate, epirubicin and cisplatin (MEC) has a positive impact on survival in patients with lymph node-positive disease. Freiha et al. (7) demonstrated that patients treated with cisplatin, methotrexate \& vinblastine (CMV) had a higher survival compared to those patients who did not receive adjuvant chemotherapy.

Michael et al. (16) evaluated 35 patients retrospectively who received either cisplatin, methotrexate, and vinblastine (CMV) or methotrexate, vinblastine, doxorubicin, and cisplatin (MVAC) and found nine episodes of febrile neutropenia (one fatal) and six episodes of thromboembolism (one fatal). The authors cautioned that the use of adjuvant chemotherapy with CMV or MVAC might cause serious toxicity. Furthermore, Pectasides et al. (18) investigated the use of Cisplatin, epirubicin and docetaxel together in transitional cell urothelial cancer and found that $53.3 \%$ patients required one dose reduction and $16.7 \%$ patients required two dose reductions for a nadir AGC $\leq 500 / \mathrm{mm}^{3}$. There were also four episodes of febrile neutropenia and sepsis occurred and no patient had a dose reduction or treatment delay for any grade 3/4 toxicity or treatment delays due to myelotoxicity. Any non-hematological toxicity was mild and infrequent. They cautioned that the response rate and toxicity of the combination of epirubicin, docetaxel and cisplatin were comparable with the methotrexate, vinblastine, doxorubicin, cisplatin (M-VAC) regimen. Unfortunately, the complication rate and ultimately safety of adjuvant chemotherapy following RC and NB reconstruction, has not been well documented in the literature.

After considering the above studies, we decided to compare the incidence of urinary tract infection, hematologic complications, and other complications in a group of patients who received a NB and adjuvant chemotherapy and one in which patients who did not for the purpose of demonstrating whether adjuvant chemotherapy is safe for patients undergoing radical cystectomy with NB. 
Hematologic complications were reported only in the group of patients who received adjuvant chemotherapy: two patients with anemia, 1 with night time fevers, 1 with anemia and neutropenic fever and 3 patients with neutropenic fever. Urinary tract infection was surprisingly higher in group-2 than group-1, but without statistically difference. For the overall complication rate, there was a statistical significant difference $(\mathrm{p}=0.015)$ demonstrated in $53 \%$ patients who received adjuvant chemotherapy compared to $23 \%$ patients who did not received adjuvant chemotherapy.

Patients, who are treated for invasive bladder cancer following $\mathrm{RC}$ with $\mathrm{NB}$, must be counseled about the toxicity of adjuvant chemotherapy, a critical component to the treatment plan. There are considerable side effects from chemotherapy, which vary, depending on the agents, dose used and the duration of treatment. Of concern always is inability of many patients to tolerate all courses of planned chemotherapy secondary to postoperative morbidity or drug side effects.

Because of the increased risk of complications, physicians may be hesitant about suggesting adjuvant chemotherapy in patients with neobladder who are at a high risk for recurrence. But the positive impact of adjuvant chemotherapy, longterm disease- free survival and the good clinical safety profile has been shown in many studies $(6,15,19)$. Furthermore, all the complications demonstrated in this study were not life threatening, transient and resolved when chemotherapy was completed. In addition, the complications reported in our study were not specific to patients with an orthotopic neobladder. In fact, most of the complications were related to adjuvant chemotherapy and can occur in other types of diversion such as ileal conduit. Manoharan et al. (20) have demonstrated that the complication rates following $\mathrm{AC}$ are similar in patients with ileal conduit and neobladder. Therefore the option of orthotopic neobladder should not be denied in bladder cancer patients at high risk for recurrent disease.

The antineoplastic agent combinations that offer the least toxic side effects with the most survival benefit have evolved during the past decade (21). Currently, the approach towards adjuvant systemic treatment has changed due to the development of more tolerable antineoplastic agents with similar efficacy. The introduction of gemcitabine, a nucleoside antimetabolite that inhibits DNA synthesis and the taxanes as chemotherapy treatment choices for bladder cancer is a promising development. Gemcitabine, as a single agent has shown an overall response rate of about $25 \%$, with a complete response rate of $9 \%$, with the combination of cisplatin has already demonstrated similar objective response and survival rates with less toxicity than M-VAC, in patients with metastatic bladder cancer $(21,22)$. At our center MVAC regimen has been largely replaced by gemcitabine and cisplatin.

There are some limitations to the interpretation of this data. This is a nonrandomized and retrospective study. Although it was a retrospective study, the strength of our series is the number (84) of high risk patients operated on by a single surgeon in the same center over 12 years. Another limitation of the study is that adjuvant chemotherapy protocols were performed by different physicians and regimens were not standard. This reflects the advances in the chemotherapeutic drugs, which resulted in regimen changes with time.

\section{CONCLUSIONS}

There was an increased incidence of complications such as hematological effects found in patients who received adjuvant chemotherapy, compared to those with neobladder alone. However symptoms were non life threatening, transient and disappeared when chemotherapy was discontinued. Adjuvant chemotherapy in neobladder patients is safe. Hence the option of orthotopic neobladder reconstruction should not be unfairly denied for bladder cancer patients, at high risk for recurrent disease, who may require adjuvant chemotherapy.

\section{ACKNOWLEDGEMENTS}

Financial support from Jackson Memorial Hospital Foundation, Mr. Harvey Chaplin, Mr. Mel Dick. 


\section{CONFLICT OF INTEREST}

\author{
None declared.
}

\section{REFERENCES}

1. National Cancer Institute (NCI) booklet (NIH Publication $\mathrm{N}^{\circ}$. 01-1559

2. Skinner DG, Studer UE, Okada K, Aso Y, Hautmann $\mathrm{H}$, Koontz W, et al.: Which patients are suitable for continent diversion or bladder substitution following cystectomy or other definitive local treatment? Int $\mathbf{J}$ Urol. 1995; 2: 105-12.

3. Parekh DJ, Gilbert WB, Koch MO, Smith JA Jr: Continent urinary reconstruction versus ileal conduit: a contemporary single-institution comparison of perioperative morbidity and mortality. Urology. 2000; 55: 852-5.

4. Dutta SC, Chang SC, Coffey CS, Smith JA Jr, Jack G, Cookson MS: Health related quality of life assessment after radical cystectomy: comparison of ileal conduit with continent orthotopic neobladder. J Urol. 2002; 168: 164-7.

5. Logothetis CJ, Johnson DE, Chong C, Dexeus FH, Ogden S, von Eschenbach A, et al.: Adjuvant chemotherapy of bladder cancer: a preliminary report. J Urol. 1988; 139: 1207-11.

6. Skinner DG, Daniels JR, Russell CA, Lieskovsky G, Boyd SD, Nichols P, et al.: The role of adjuvant chemotherapy following cystectomy for invasive bladder cancer: a prospective comparative trial. J Urol. 1991; 145: 459-64; discussion 464-7.

7. Freiha F, Reese J, Torti FM: A randomized trial of radical cystectomy versus radical cystectomy plus cisplatin, vinblastine and methotrexate chemotherapy for muscle invasive bladder cancer. J Urol. 1996; 155: 495-9.

8. Herr HW: Does current chemotherapy improve survival of patients with invasive bladder cancer? Urology. 1997; 49: 309-12.

9. Hautmann RE, Egghart G, Frohneberg D, Miller K: The ileal neobladder. J Urol. 1988; 139: 39-42.

10. Studer UE, Ackermann D, Casanova GA, Zingg EJ: Three years' experience with an ileal low pressure bladder substitute. Br J Urol. 1989; 63: 43-52.

11. Gerharz EW, Weingartner K, Dopatka T, Kohl UN, Basler HD, Riedmiller HN: Quality of life after cystectomy and urinary diversion: results of a retrospective interdisciplinary study. J Urol. 1997; 158: 778-85.

12. McGuire MS, Grimaldi G, Grotas J, Russo P: The type of urinary diversion after radical cystectomy significantly impacts on the patient's quality of life. Ann Surg Oncol. 2000; 7: 4-8.

13. Wullt B, Holst E, Steven K, Carstensen J, Pedersen J, Gustafsson E, et al.: Microbial flora in ileal and colonic neobladders. Eur Urol. 2004; 45: 233-9.

14. Wood DP Jr, Bianco FJ Jr, Pontes JE, Heath MA, DaJusta D: Incidence and significance of positive urine cultures in patients with an orthotopic neobladder. $\mathbf{J}$ Urol. 2003; 169: 2196-9.

15. Stockle M, Meyenburg W, Wellek S, Voges GE, Rossmann M, Gertenbach U, et al.: Adjuvant polychemotherapy of nonorgan-confined bladder cancer after radical cystectomy revisited: long-term results of a controlled prospective study and further clinical experience. J Urol. 1995; 153: 47-52.

16. Michael M, Tannock IF, Czaykowski PM, Moore MJ: Adjuvant chemotherapy for high-risk urothelial transitional cell carcinoma: the Princess Margaret Hospital experience. Br J Urol. 1998; 82: 366-72.

17. Suzuki S, Shinohara N, Harabayashi T, Sato S, Abe T, Koyanagi T: Impact of adjuvant systemic chemotherapy on postoperative survival in patients with high-risk urothelial cancer. Int J Urol. 2004; 11: 456-60.

18. Pectasides D, Visvikis A, Aspropotamitis A, Halikia A, Karvounis N, Dimitriadis M, et al.: Chemotherapy with cisplatin, epirubicin and docetaxel in transitional cell urothelial cancer. Phase II trial. Eur J Cancer. 2000; 36: 74-9.

19. Stockle M, Wellek S, Meyenburg W, Voges GE, Fischer U, Gertenbach U, et al.: Radical cystectomy with or without adjuvant polychemotherapy for non-organconfined transitional cell carcinoma of the urinary bladder: prognostic impact of lymph node involvement. Urology. 1996; 48: 868-75.

20. Manoharan M, Reyes MA, Kava BR, Singal R, Kim SS, Soloway MS: Is adjuvant chemotherapy for bladder cancer safer in patients with an ileal conduit than a neobladder? BJU Int. 2005; 96: 1286-9.

21. von der Maase H, Hansen SW, Roberts JT, Dogliotti L, Oliver T, Moore MJ, et al.: Gemcitabine and cisplatin versus methotrexate, vinblastine, doxorubicin, and cisplatin in advanced or metastatic bladder cancer: results of a large, randomized, multinational, multicenter, phase III study. J Clin Oncol. 2000; 18: 3068-77. 
22. Moore MJ, Tannock IF, Ernst DS, Huan S, Murray N: Gemcitabine: a promising new agent in the treatment

Correspondence address:

Dr. M. Manoharan

Department of Urology

Miller School of Medicine University of Miami

P.O. Box 016960 (M814)

Miami, Florida, 33101, USA

Fax: +1 305 243-4653

E-mail: mmanoharan@med.miami.edu

\section{EDITORIAL COMMENT}

A recent meta-analysis addressing the value of adjuvant chemotherapy in bladder cancer has shown an absolute benefit of $11 \%$ and $16 \%$ in the 5 year overall survival and disease free survival in favor of adjuvant chemotherapy respectively. Unfortunately, all randomized studies present serious methodological problems making these results less reliable (1).

Despite the retrospective design of the present study it brings the message that hematological side effects of chemotherapy are mild and tolerable. of advanced urothelial cancer. J Clin Oncol. 1997; 15: 3441-5.

Accepted after revision:

June 30, 2006
Furthermore, in the present days with the advent of new drugs with less toxicity we can whiteness better tolerance of the patients.

\section{REFERENCE}

1. Ruggeri EM, Giannarelli D, Bria E, Carlini P, Felici A, Nelli F, et al.: Adjuvant chemotherapy in muscleinvasive bladder carcinoma: a pooled analysis from phase III studies. Cancer. 2006; 106: 783-8.

Dr. Marcos F. Dall'Oglio Division of Urology University of São Paulo, USP São Paulo, SP, Brazil E-mail:marcosdallogliouro@terra.com.br 